\title{
WELL-POSEDNESS \\ FOR THE SCHRÖDINGER-KORTEWEG-DE VRIES SYSTEM
}

\author{
A. J. CORCHO AND F. LINARES
}

\begin{abstract}
We study well-posedness of the Cauchy problem associated to the Schrödinger-Korteweg-de Vries system. We obtain local well-posedness for weak initial data, where the best result obtained is for data in the Sobolev space $L^{2}(\mathbb{R}) \times H^{-\frac{3}{4}+}$. This result implies in particular the global well-posedness in the energy space $H^{1}(\mathbb{R}) \times H^{1}(\mathbb{R})$. Both results considerably improve the previous ones by Bekiranov, Ogawa and Ponce (1997), Guo and Miao (1999), and Tsutsumi (1993).
\end{abstract}

\section{INTRODUCTION}

This paper is concerned with the Initial Value Problem (IVP) for the SchrödingerKorteweg-de Vries (NLS-KdV) system

$$
\left\{\begin{array}{l}
i \partial_{t} u+\partial_{x}^{2} u=\alpha u v+\beta|u|^{2} u, \quad t, x \in \mathbb{R}, \\
\partial_{t} v+\partial_{x}^{3} v+\frac{1}{2} \partial_{x}\left(v^{2}\right)=\gamma \partial_{x}\left(|u|^{2}\right), \\
u(x, 0)=u_{0}(x), v(x, 0)=v_{0}(x),
\end{array}\right.
$$

where $u=u(x, t)$ is a complex-valued function, $v=v(x, t)$ is a real-valued function and $\alpha, \beta, \gamma$ are real constants.

This system governs the interactions between short-wave, $u=u(x, t)$, and longwave, $v=v(x, t)$, and arises in fluid mechanics as well as plasma physics. The case $\beta=0$ appears in the study of resonant interaction between short and long capillary-gravity waves on water of uniform finite depth, in plasma physics and in a diatomic lattice system. See [8], [1], [12] and [17] for these applications.

In [3] it was showed that the NLS-KdV system is not completely integrable, therefore, the solvability of (1.1) is dependent upon the method of the evolution equations.

M. Tsutsumi [19] showed that for data $\left(u_{0}, v_{0}\right) \in H^{s+\frac{1}{2}}(\mathbb{R}) \times H^{s}(\mathbb{R})$ with $s \in \mathbb{Z}^{+}$ the coupled system (1.1) is globally well-posed in $H^{s+\frac{1}{2}}(\mathbb{R}) \times H^{s}(\mathbb{R})$ using the

Received by the editors February 4, 2005.

2000 Mathematics Subject Classification. Primary 35Q55, 35Q60, 35B65.

Key words and phrases. Well-posedness, Schrödinger-Korteweg-de Vries system.

The first author was supported by CNPq and FAPEAL, Brazil.

The second author was partially supported by CNPq, Brazil.

(C)2007 American Mathematical Society Reverts to public domain 28 years from publication 
following conserved functionals for the flow defined by (1.1): namely,

$$
\begin{gathered}
\mathcal{M}(t):=\int_{-\infty}^{+\infty}|u|^{2} d x=\mathcal{M}(0), \\
\mathcal{Q}(t):=\int_{-\infty}^{+\infty}\left\{\alpha v^{2}+2 \gamma \operatorname{Im}\left(u \overline{\partial_{x} u}\right)\right\} d x=\mathcal{Q}(0),
\end{gathered}
$$

and

$$
\mathcal{E}(t):=\int_{-\infty}^{+\infty}\left\{\alpha \gamma v|u|^{2}-\frac{\alpha}{6} v^{3}+\frac{\beta \gamma}{2}|u|^{4}+\frac{\alpha}{2}\left|\partial_{x} v\right|^{2}+\gamma\left|\partial_{x} u\right|^{2}\right\} d x=\mathcal{E}(0) .
$$

The resonant case $(\beta=0)$ was considered by Guo and Miao [10], and they have proved global well-posedness for data in $H^{s}(\mathbb{R}) \times H^{s}(\mathbb{R})$ with $s \in \mathbb{Z}^{+}$. We consider $\alpha, \beta$ and $\gamma$ different from zero.

For the single nonlinear Schrödinger (NLS) equation with cubic term $\left(|u|^{2} u\right)$, Y. Tsutsumi [18, established local and global well-posedness for data in $L^{2}(\mathbb{R})$. On the other hand, for the Korteweg-de Vries (KdV) equation Kenig, Ponce and Vega [14 have proved local well-posedness for data in $H^{s}(\mathbb{R}), s>-\frac{3}{4}$. See also [4] and [13. for other local well-posedness results in Sobolev spaces with negative exponents.

By using the scaling and Galilean invariance with the special soliton solutions, it was proved by Kenig, Ponce and Vega [16 that the focusing cubic NLS equation is not locally-well posed (ill-posed) below $L^{2}(\mathbb{R})$ in the sense that the data-solution map is not uniformly continuous. Recently, Christ, Colliander and Tao [5, 6] have obtained similar results for the KdV and defocusing NLS equations. More precisely, they have proved that the data-solution map fails to be uniformly continuous with respect to the $H^{s}$-norm for $s \in\left[-1,-\frac{3}{4}\right)$ in the case of $\mathrm{KdV}$ equation and for $s<0$ in the case of the cubic defocusing NLS equation.

The coupled system (1.1) is more difficult to handle in the same spaces than in the single equation case. It is due to the antisymmetric nature of the characteristics of each linear part. In [1] Bekiranov, Ogawa and Ponce showed that the coupled system (1.1) is locally well-posed in $H^{s}(\mathbb{R}) \times H^{s-\frac{1}{2}}(\mathbb{R})$ with $s \geq 0$. These results include and extend the previous ones obtained in 19 . Moreover, we note that the best result in 1 is in $L^{2}(\mathbb{R}) \times H^{-\frac{1}{2}}(\mathbb{R})$, and to obtain this, the authors used the Fourier restriction norm method introduced by Bourgain in [4] to study the NLS and KdV equations. This method was extensively improved by Kenig, Ponce and Vega in [13, [14, and [15] to establish their results for the KdV and NLS equations.

Taking into account the sharp local well-posedness results obtained for the NLS and $\mathrm{KdV}$ equations it is natural to ask whether the NLS-KdV system is locally wellposed in $L^{2}(\mathbb{R}) \times H^{-\frac{3}{4}+}(\mathbb{R})$. In this work we answer affirmatively this question. Indeed we obtain local well-posedness for weak initial data $\left(u_{0}, v_{0}\right) \in H^{k}(\mathbb{R}) \times H^{s}(\mathbb{R})$ for various values of $k$ and $s$, where the lowest admissible values are $k=0$ and $s=-\frac{3}{4}+\delta$ with $0<\delta \leq \frac{1}{4}$. The scheme of proof used to obtain our results is in the same spirit as the one implemented by Ginibre, Y. Tsutsumi and Velo 9] to establish their results for the Zakharov system. Moreover, our results cover the case $k=s=1$, and hence we can use the conserved quantities (1.2)-(1.4) to obtain global well-posedness in the energy space $H^{1}(\mathbb{R}) \times H^{1}(\mathbb{R})$, improving previous results obtained in [10] and [19]. 
Before stating the results we will give some notation. Let $U(t)=e^{i t \partial_{x}^{2}}$ and $V(t)=e^{-t \partial_{x}^{3}}$ be the unitary groups associated with the linear Schrödinger and the linear $\mathrm{KdV}$ equations, respectively. Now we introduce the function spaces for constructing the local solutions. For $k, s \in \mathbb{R}$ and $b \in(0,1)$ we let $X^{k, b}$ and $Y^{s, b}$ be the completion of $\mathcal{S}\left(\mathbb{R}^{2}\right)$ with respect to norms

$$
\begin{aligned}
\|f\|_{X^{k, b}} & =\left(\iint\langle\xi\rangle^{2 k}\left\langle\tau+\xi^{2}\right\rangle^{2 b}|\widehat{f}(\xi, \tau)|^{2} d \tau d \xi\right)^{\frac{1}{2}} \\
& =\|U(-t) f\|_{H_{t}^{b}\left(\mathbb{R}, H_{x}^{k}\right)} \\
\|g\|_{Y^{s, b}} & =\left(\iint\langle\xi\rangle^{2 s}\left\langle\tau-\xi^{3}\right\rangle^{2 b}|\widehat{g}(\xi, \tau)|^{2} d \tau d \xi\right)^{\frac{1}{2}} \\
& =\|V(-t) g\|_{H_{t}^{b}\left(\mathbb{R}, H_{x}^{s}\right)}
\end{aligned}
$$

where $\langle\cdot\rangle=1+|\cdot|$ and $\widehat{f}$ denotes the Fourier transform of $f$ in both $x$ and $t$ variables

$$
\widehat{f}(\tau, \xi)=(2 \pi)^{-1} \iint_{\mathbb{R}^{2}} e^{-i t \tau-i x \xi} f(x, t) d t d x .
$$

In what follows $\psi$ denotes a cut-off function in $C_{0}^{\infty}(\mathbb{R})$ such that

$$
\psi(t)= \begin{cases}1 & \text { if }|t| \leq 1 \\ 0 & \text { if }|t| \geq 2\end{cases}
$$

$0 \leq \psi(t) \leq 1$, and let $\psi_{T}(t):=\psi\left(\frac{t}{T}\right)$ for $0 \leq T \leq 1$. Finally, we remark that various constants are denoted by $C$ and that $\lambda \pm$ denotes a number slightly larger, respectively smaller, than $\lambda$.

Now we give the statements of our main results concerning well-posedness for the IVP (1.1).

The local well-posedness result is as follows.

Theorem 1.1. Let $k \geq 0$ and $s>-\frac{3}{4}$. Then for any $\left(u_{0}, v_{0}\right) \in H^{k}(\mathbb{R}) \times H^{s}(\mathbb{R})$ provided:

(i) $k-1 \leq s \leq 2 k-\frac{1}{2}$ for $k \in\left[0, \frac{1}{2}\right]$,

(ii) $k-1 \leq s<k+\frac{1}{2}$ for $k \in\left(\frac{1}{2}, \infty\right)$,

there exist a positive time $T=T\left(\left\|u_{0}\right\|_{H^{k}},\left\|v_{0}\right\|_{H^{s}}\right)$ and a unique solution $(u(t), v(t))$ of the initial value problem (1.1), satisfying

$$
\begin{array}{rll}
\psi_{T}(t) u \in X^{k, \frac{1}{2}+} \quad \text { and } & \psi_{T}(t) v \in Y^{s, \frac{1}{2}+}, \\
u \in C\left([0, T] ; H^{k}(\mathbb{R})\right) & \text { and } \quad v \in C\left([0, T] ; H^{s}(\mathbb{R})\right) .
\end{array}
$$

Moreover, the map $\left(u_{0}, v_{0}\right) \longmapsto(u(t), v(t))$ is locally Lipschitz from $H^{k}(\mathbb{R}) \times$ $H^{s}(\mathbb{R})$ into $C\left([0, T] ; H^{k}(\mathbb{R}) \times H^{s}(\mathbb{R})\right)$.

Remark 1.1. The key point in the proof of Theorem 1.1 is the deduction and use of new bilinear estimates in Section 3 for the coupling terms in system (1.1). Note that these bilinear estimates are sharper than the ones obtained in [1].

Concerning global well-posedness we have the following result.

Theorem 1.2. Let $\alpha, \beta, \gamma \in \mathbb{R}$ such that $\alpha \gamma>0$ and $\left(u_{0}, v_{0}\right) \in H^{1}(\mathbb{R}) \times H^{1}(\mathbb{R})$. Then, the unique solution provided by Theorem 1.1 can be extended for any time $T>0$. 


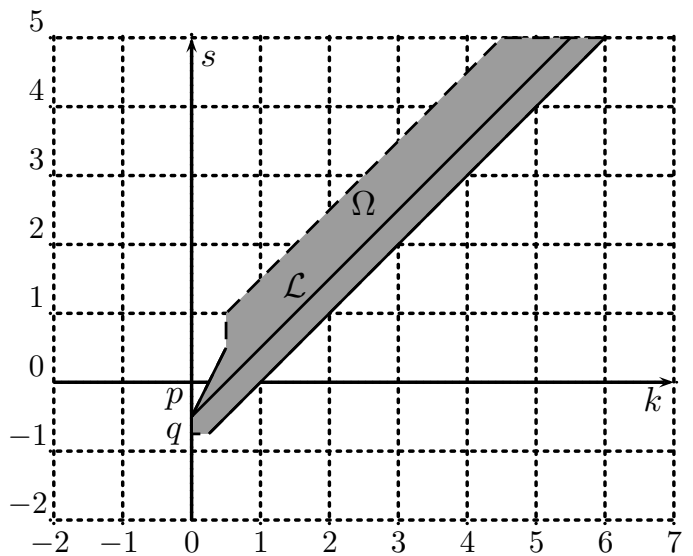

FiguRE 1. Well-posedness results for the NLS-KdV system. The region $\Omega$ contains indices $(k, s)$ for which local well-posedness is achieved in Theorem 1.1. This region contains the line $\mathcal{L}=$ $\left\{(k, s) \in \mathbb{R}^{2} ; k \geq 0\right.$ and $\left.k-s=\frac{1}{2}\right\}$ corresponding to the results proved by Bekiranov, Ogawa and Ponce in [1. Moreover, the best results obtained in Theorem 1.1 are represented in the line segment $\overline{p q}=\left\{(0, s) \in \mathbb{R}^{2} ;-\frac{3}{4}<s \leq-\frac{1}{2}\right\}$, and these improve the ones given in [1].

Remark 1.2. We note that if $\beta \neq 0$ the lowest regularity assumption admissible for the data, namely $k=0$ and $s=-\frac{3}{4}+$, is optimal in the framework of the method that we use to prove Theorem 1.1 in the sense that the estimates (2.2) and (2.3) fail below $k=0$ and $s=-\frac{3}{4}$, respectively. See [5, [6] and [16].

The paper is organized as follows. In Section 2 we state a series of results needed in the remainder of this work. The new bilinear estimates are established in Section 3. The main result is proved in Section 4. Finally, in Section 5, we show Theorem 1.2 .

\section{Preliminary Results}

We consider the equation of the form

$$
i \partial_{t} \omega-\phi\left(-i \partial_{x}\right) \omega=F(\omega),
$$

where $\phi$ is a measurable real-valued function and $F$ some nonlinear function.

The Cauchy problem for (2.1) with initial data $\omega(0)=\omega_{0}$ is rewritten as the integral equation

$$
\omega(t)=W_{\phi}(t) \omega_{0}-i \int_{0}^{t} W_{\phi}\left(t-t^{\prime}\right) F\left(\omega\left(t^{\prime}\right)\right) d t^{\prime},
$$

where $W_{\phi}(t)=e^{-i t \phi\left(-i \partial_{x}\right)}$ is the unitary group that solves the linear part of (2.1). 
Let $X^{s, b}(\phi)$ be the completion of $\mathcal{S}\left(\mathbb{R}^{2}\right)$ with respect to the norm

$$
\begin{aligned}
\|f\|_{X^{s, b}(\phi)} & \equiv\left\|W_{\phi}(-t) f\right\|_{H_{t}^{b}\left(\mathbb{R}, H_{x}^{s}\right)} \\
& =\left\|\langle\xi\rangle^{s}\langle\tau\rangle^{b} \mathcal{F}\left(e^{i t \phi\left(-i \partial_{x}\right)} f\right)(\tau, \xi)\right\|_{L_{\tau}^{2} L_{\xi}^{2}} \\
& =\left\|\langle\xi\rangle^{s}\langle\tau+\phi(\xi)\rangle^{b} \widehat{f}(\tau, \xi)\right\|_{L_{\tau}^{2} L_{\xi}^{2}} .
\end{aligned}
$$

The following lemma has been proved while establishing the local well-posedness of the Zakharov system by Ginibre, Y. Tsutsumi and Velo in [9].

Lemma 2.1. Let $-\frac{1}{2}<b^{\prime} \leq 0 \leq b \leq b^{\prime}+1$ and $T \in[0,1]$. Then for $F \in X^{s, b^{\prime}}(\phi)$ we have

$$
\begin{aligned}
& \left\|\psi_{1}(t) W_{\phi}(t) \omega_{0}\right\|_{X^{s, b}(\phi)} \leq C\left\|\omega_{0}\right\|_{H^{s}}, \\
& \left\|\psi_{T}(t) \int_{0}^{t} W_{\phi}\left(t-t^{\prime}\right) F\left(t^{\prime}, \cdot\right) d t^{\prime}\right\|_{X^{s, b}(\phi)} \leq C T^{1-b+b^{\prime}}\|F\|_{X^{s, b^{\prime}}(\phi)} .
\end{aligned}
$$

Proof. See Lemma 2.1 in $[9$.

In our case we shall use the spaces $X^{s, b}(\phi)$ for the phase functions $\phi_{1}(\xi)=\xi^{2}$ and $\phi_{2}(\xi)=-\xi^{3}$. Indeed we can rewrite the system (1.1) in the form

$$
\left\{\begin{array}{l}
i \partial_{t} u-\phi_{1}\left(-i \partial_{x}\right) u=\alpha u v+\beta|u|^{2} u \\
i \partial_{t} v-\phi_{2}\left(-i \partial_{x}\right) v=i \gamma \partial_{x}\left(|u|^{2}\right)-i \frac{1}{2} \partial_{x}\left(v^{2}\right) .
\end{array}\right.
$$

Then we have

$$
X^{k, b}\left(\phi_{1}\right)=X^{k, b}, \quad W_{\phi_{1}}=U(t)=e^{i t \partial_{x}^{2}},
$$

and

$$
X^{s, b}\left(\phi_{2}\right)=Y^{s, b}, \quad W_{\phi_{2}}=V(t)=e^{-t \partial_{x}^{3}},
$$

where $U(t)$ and $V(t)$ are the linear Schrödinger and Airy unitary groups, respectively.

For a given time interval $I$ we define

$$
\|f\|_{X_{I}^{k, b}}=\inf _{\tilde{f}_{\mid I}=f}\|\tilde{f}\|_{X^{k, b}} \quad \text { and } \quad\|f\|_{Y_{I}^{s, b}}=\inf _{\tilde{f}_{\mid I}=f}\|\tilde{f}\|_{Y^{s, b}} .
$$

If $b>1 / 2$, the Sobolev lemma implies

$$
X_{I}^{k, b} \hookrightarrow C\left(I ; H_{x}^{k}(\mathbb{R})\right) \quad \text { and } \quad Y_{I}^{s, b} \hookrightarrow C\left(I ; H_{x}^{s}(\mathbb{R})\right) .
$$

Now we give estimates for the nonlinear terms that are needed in the proof of Theorem 1.1. We begin with the cubic nonlinear term.

Lemma 2.2. Let $u, \tilde{u} \in X^{k, b}$ with $\frac{1}{2}<b<1$ and $k \geq 0$. Then for $a \geq 0$ we have that

$$
\begin{gathered}
\left\||u|^{2} u\right\|_{X^{k,-a}} \leq C\|u\|_{X^{k, b}}^{3}, \\
\left\||u|^{2} u-|\tilde{u}|^{2} \tilde{u}\right\|_{X^{k,-a}} \leq C\left(\|u\|_{X^{k, b}}^{2}+\|\tilde{u}\|_{X^{k, b}}^{2}\right)\|u-\tilde{u}\|_{X^{k, b}} .
\end{gathered}
$$

Proof. See Lemma 3.1 in [2].

The following lemma is due to Kenig, Ponce and Vega in [14]. 
Lemma 2.3. Let $v, \tilde{v} \in Y^{s, b}$. Then there exists $C>0$ such that

$$
\begin{gathered}
\left\|\partial_{x}\left(v^{2}\right)\right\|_{Y^{s,-a}} \leq C\|v\|_{Y^{s, b}}^{2}, \\
\left\|\partial_{x}\left(v^{2}\right)-\partial_{x}\left(\tilde{v}^{2}\right)\right\|_{Y^{s,-a}} \leq C\left(\|v\|_{Y^{s, b}}+\|\tilde{v}\|_{Y^{s, b}}\right)\|v-\tilde{v}\|_{Y^{s, b}},
\end{gathered}
$$

hold in the following cases:

(i) $1-b-\min \left\{-\frac{2 s+1}{2}, \frac{4 s+3}{12}\right\} \leq a<\frac{1}{2}<b \leq 1-a$, for $s \in\left(-\frac{3}{4},-\frac{1}{2}\right)$,

(ii) $\frac{5}{12}<a<\frac{1}{2}<b<\frac{7}{12}$, for $s \in\left[-\frac{1}{2}, 0\right)$,

(iii) $\frac{1}{4} \leq a<\frac{1}{2}<b \leq 1-a$, for $s \in[0, \infty)$.

Proof. See references [13] and [14].

We finish this section with the following inequalities which will be used to estimate the nonlinear terms in Section 3 .

Lemma 2.4. For $p, q>0, r=\min \{p, q\}$ with $p+q>1+r, s>1$ and $t>\frac{1}{3}$, there exists $C>0$ such that

$$
\begin{gathered}
\int_{-\infty}^{\infty} \frac{d x}{(\mu+|x-\alpha|)^{p}(\mu+|x-\beta|)^{q}} \leq C \frac{\mu^{-(p+q-1-r)}}{(\mu+|\alpha-\beta|)^{r}}, \quad \text { for } \quad \mu>0 \\
\int_{-\infty}^{\infty} \frac{d x}{\langle\alpha x-\beta\rangle^{s}} \leq \frac{C}{|\alpha|}, \quad \text { for } \quad \alpha \neq 0 \\
\int_{-\infty}^{\infty} \frac{d x}{\left\langle\alpha_{0}+\alpha_{1} x+\alpha_{2} x^{2}+x^{3}\right\rangle^{t}} \leq C .
\end{gathered}
$$

Proof. The inequalities (2.11) with $\mu=1$ and (2.12) follow from simple calculus. They are given in [1] and 14]. We prove (2.11) for any $\mu>0$ as follows:

$$
\begin{gathered}
\int_{-\infty}^{\infty} \frac{d x}{(\mu+|x-\alpha|)^{p}(\mu+|x-\beta|)^{q}}=\int_{-\infty}^{\infty} \frac{\mu d y}{(\mu+|\mu y-\alpha|)^{p}(\mu+|\mu y-\beta|)^{q}} \\
\quad=\mu^{1-p-q} \int_{-\infty}^{\infty} \frac{d y}{\left(1+\left|y-\mu^{-1} \alpha\right|\right)^{p}\left(1+\left|y-\mu^{-1} \beta\right|\right)^{q}} \\
\quad \leq \mu^{1-p-q} \frac{C}{\left(1+\mu^{-1}|\alpha-\beta|\right)^{r}}=C \frac{\mu^{1+r-p-q}}{(\mu+|\alpha-\beta|)^{r}},
\end{gathered}
$$

where in the last inequality we have used the case $\mu=1$.

Finally, for the proof of (2.13) we can see Lemma 2.5 in [1].

We use the generalization of (2.11) with $\mu>0$ in the proof of Lemma 3.2 in the next section.

\section{Bilinear estimates For the COUPLing terms}

Next we prove new bilinear estimates for the interaction terms. Our results improve the estimates given by Bekiranov, Ogawa and Ponce for these terms in 1 .

Lemma 3.1. Given $k \geq 0, \frac{1}{6} \leq a<\frac{1}{2}<b$ and $k-s \leq \min \{1,3 a\}$, there exists a positive constant $C$ depending only on the parameters $a, b, k$ and $s$ such that for any $u, \tilde{u} \in X^{k, b}$ and $v, \tilde{v} \in Y^{s, b}$

$$
\begin{gathered}
\|u v\|_{X^{k,-a}} \leq C\|u\|_{X^{k, b}}\|v\|_{Y^{s, b}}, \\
\|u v-\tilde{u} \tilde{v}\|_{X^{k,-a}} \leq C\left(\|u-\tilde{u}\|_{X^{k, b}}\|v\|_{Y^{s, b}}+\|\tilde{u}\|_{X^{k, b}}\|v-\tilde{v}\|_{Y^{s, b}}\right) .
\end{gathered}
$$


Lemma 3.2. Let $u_{j}, \tilde{u}_{j} \in X^{k, b}, j=1,2$, with $b>\frac{1}{2}$ and $k \geq 0$. Then for $0 \leq a \leq$ $b$ there is a positive constant depending only on the parameters $a, b, k$ and $s$ such that

$$
\begin{gathered}
\left\|\partial_{x}\left(u_{1} \bar{u}_{2}\right)\right\|_{Y^{s,-a}} \leq C\left\|u_{1}\right\|_{X^{k, b}}\left\|u_{2}\right\|_{X^{k, b}}, \\
\left.\left\|\partial_{x}\left(\left|u_{1}\right|^{2}\right)-\partial_{x}\left(\left|\tilde{u}_{1}\right|^{2}\right)\right\|_{Y^{s,-a}} \leq C\left(\left\|u_{1}\right\|_{X^{k, b}}+\left\|\tilde{u}_{1}\right\|_{X^{k, b}}\right)\left\|u_{1}-\tilde{u}_{1}\right\|_{X^{k, b}}\right),
\end{gathered}
$$

hold in the following cases:

(i) $s-2 k \leq \min \left\{3 a-2 b-\frac{1}{2},-\frac{1}{2}\right\}$, for $k \in\left[0, \frac{1}{2}\right]$,

(ii) $s-k \leq 3 a-b-\frac{1}{2}$, for $k \in\left(\frac{1}{2}, \infty\right)$ and $b \in\left(\frac{1}{2}, k\right]$.

Remark 3.1. The estimates (3.2) and (3.4) can be deduced from the same argument used to show estimates (3.1) and (3.3), respectively.

Now we give the proofs of the statements above. We follow closely the argument in [1].

\subsection{Proof of Lemma 3.1. We let}

$$
f(\tau, \xi)=\left\langle\tau+\xi^{2}\right\rangle^{b}\langle\xi\rangle^{k} \widehat{u}(\xi, \tau) \text { and } g(\tau, \xi)=\left\langle\tau-\xi^{3}\right\rangle^{b}\langle\xi\rangle^{s} \widehat{v}(\xi, \tau)
$$

and denote

to obtain the following:

$$
I\left(\varphi_{1}, \varphi_{2}\right)=\iint_{\mathbb{R}^{2}} \varphi_{1} \bar{\varphi}_{2} d x d y
$$

$$
\begin{aligned}
\|u v\|_{X^{k,-a}} & =\left\|\left\langle\tau+\xi^{2}\right\rangle^{-a}\langle\xi\rangle^{k} \widehat{u v}(\xi, \tau)\right\|_{L_{\tau}^{2} L_{\xi}^{2}} \\
& =\left\|\frac{\langle\xi\rangle^{k}}{\left\langle\tau+\xi^{2}\right\rangle^{a}} \widehat{u} * \widehat{v}(\xi, \tau)\right\|_{L_{\tau}^{2} L_{\xi}^{2}} \\
& =\sup _{\|\varphi\|_{L_{\tau, \xi}^{2} \leq 1}}\left|I\left(\frac{\langle\xi\rangle^{k}}{\left\langle\tau+\xi^{2}\right\rangle^{a}} \widehat{u} * \widehat{v}, \varphi\right)\right| \\
& :=\sup _{\|\varphi\|_{L_{\tau, \xi}^{2} \leq 1} \leq W(u, v, \varphi) \mid,} \mid
\end{aligned}
$$

where

$$
\begin{aligned}
& W(u, v, \varphi)=I\left(\frac{\langle\xi\rangle^{k}}{\left\langle\tau+\xi^{2}\right\rangle^{a}}\left\{\frac{f}{\left\langle\tau+\xi^{2}\right\rangle^{b}\langle\xi\rangle^{k}} * \frac{g}{\left\langle\tau-\xi^{3}\right\rangle^{b}\langle\xi\rangle^{s}}\right\}, \varphi\right) \\
& =\iiint \int_{\mathbb{R}^{4}} \frac{\left\langle\tau+\xi^{2}\right\rangle^{-a}\langle\xi\rangle^{k} g\left(\xi_{1}, \tau_{1}\right) f\left(\xi-\xi_{1}, \tau-\tau_{1}\right) \bar{\varphi}(\tau, \xi)}{\left\langle\tau_{1}-\xi_{1}^{3}\right\rangle^{b}\left\langle\xi_{1}\right\rangle^{s}\left\langle\tau-\tau_{1}+\left(\xi-\xi_{1}\right)^{2}\right\rangle^{b}\left\langle\xi-\xi_{1}\right\rangle^{k}} d \tau_{1} d \xi_{1} d \tau d \xi \\
& =\iiint \int_{\mathcal{R}_{1}}+\iiint \int_{\mathcal{R}_{2}}+\iiint \int_{\mathcal{R}_{3}} \equiv W_{1}+W_{2}+W_{3},
\end{aligned}
$$

with $\mathbb{R}^{4}=\mathcal{R}_{1} \cup \mathcal{R}_{2} \cup \mathcal{R}_{3}$ and $\mathcal{R}_{i}, i=1,2,3$, defined as follows.

First we split $\mathbb{R}^{4}$ into three regions $\mathcal{A}, \mathcal{B}$ and $\mathcal{C}$ :

$$
\begin{aligned}
& \mathcal{A}=\left\{\left(\xi, \xi_{1}, \tau, \tau_{1}\right) \in \mathbb{R}^{4} ;\left|\xi_{1}\right| \leq 2\right\}, \\
& \mathcal{B}=\left\{\left(\xi, \xi_{1}, \tau, \tau_{1}\right) \in \mathbb{R}^{4} ;\left|\xi_{1}\right|>2 \text { and }\left|3 \xi_{1}^{2}-2 \xi_{1}+2 \xi\right| \geq \frac{1}{2}\left|\xi_{1}\right|^{2}\right\}, \\
& \mathcal{C}=\left\{\left(\xi, \xi_{1}, \tau, \tau_{1}\right) \in \mathbb{R}^{4} ;\left|\xi_{1}\right|>2 \text { and }\left|\xi_{1}^{2}-\xi_{1}+2 \xi\right| \geq \frac{1}{2}\left|\xi_{1}\right|^{2}\right\} .
\end{aligned}
$$

Since

$\mathcal{D}=\left\{\left(\xi, \xi_{1}, \tau, \tau_{1}\right) \in \mathbb{R}^{4} ;\left|3 \xi_{1}^{2}-2 \xi_{1}+2 \xi\right|<\frac{1}{2}\left|\xi_{1}\right|^{2},\left|\xi_{1}^{2}-\xi_{1}+2 \xi\right|<\frac{1}{2}\left|\xi_{1}\right|^{2}\right.$ and $\left.\left|\xi_{1}\right|>2\right\}$ 
is empty, we have that $\mathbb{R}^{4}=\mathcal{A} \cup \mathcal{B} \cup \mathcal{C}$. Indeed if $\left(\xi, \xi_{1}, \tau, \tau_{1}\right) \in \mathcal{D}$, then

$$
\left|\xi_{1}\right|^{2}>\left|3 \xi_{1}^{2}-2 \xi_{1}+2 \xi\right|+\left|\xi_{1}^{2}-\xi_{1}+2 \xi\right| \geq\left|2 \xi_{1}^{2}-\xi_{1}\right|=\left|\xi_{1}\right|\left|2 \xi_{1}-1\right|
$$

and hence $\left|\xi_{1}\right|>\left|2 \xi_{1}-1\right|$, which is a contradiction with the condition $\left|\xi_{1}\right|>2$.

Note that for any point in $\mathcal{C}$ we have that

$$
\left|\tau+\xi^{2}\right|+\left|\tau_{1}-\xi_{1}^{3}\right|+\left|\tau-\tau_{1}+\left(\xi-\xi_{1}\right)^{2}\right| \geq\left|\xi_{1}^{3}-\xi_{1}^{2}+2 \xi \xi_{1}\right| \geq \frac{1}{2}\left|\xi_{1}\right|^{3} .
$$

Now we separate $\mathcal{C}$ into three parts:

$$
\begin{aligned}
& \mathcal{C}_{1}=\left\{\left(\xi, \xi_{1}, \tau, \tau_{1}\right) \in \mathcal{C} ;\left|\tau_{1}-\xi_{1}^{3}\right|,\left|\tau-\tau_{1}+\left(\xi-\xi_{1}\right)^{2}\right| \leq\left|\tau+\xi^{2}\right|\right\}, \\
& \mathcal{C}_{2}=\left\{\left(\xi, \xi_{1}, \tau, \tau_{1}\right) \in \mathcal{C} ;\left|\tau+\xi^{2}\right|,\left|\tau-\tau_{1}+\left(\xi-\xi_{1}\right)^{2}\right| \leq\left|\tau_{1}-\xi_{1}^{3}\right|\right\}, \\
& \mathcal{C}_{3}=\left\{\left(\xi, \xi_{1}, \tau, \tau_{1}\right) \in \mathcal{C} ;\left|\tau+\xi^{2}\right|,\left|\tau_{1}-\xi_{1}^{3}\right| \leq\left|\tau-\tau_{1}+\left(\xi-\xi_{1}\right)^{2}\right|\right\},
\end{aligned}
$$

so that one of $\left|\tau+\xi^{2}\right|,\left|\tau_{1}-\xi_{1}^{3}\right|$ or $\left|\tau-\tau_{1}+\left(\xi-\xi_{1}\right)^{2}\right|$ is larger than $\frac{1}{6}\left|\xi_{1}\right|^{3}$.

We can now define the sets $\mathcal{R}_{i}, i=1,2,3$, as follows:

$$
\mathcal{R}_{1}=\mathcal{A} \cup \mathcal{B} \cup \mathcal{C}_{1}, \quad \mathcal{R}_{2}=\mathcal{C}_{2}, \quad \mathcal{R}_{3}=\mathcal{C}_{3},
$$

and it is clear that $\mathbb{R}^{4}=\mathcal{R}_{1} \cup \mathcal{R}_{2} \cup \mathcal{R}_{3}$.

In what follows $\chi_{\mathcal{R}}$ denotes the characteristic function of the set $\mathcal{R}$.

Now to estimate $W_{1}$ we integrate over $\tau_{1}$ and $\xi_{1}$ first, and then we use the Cauchy-Schwarz and Hölder inequalities to obtain

$$
\begin{aligned}
& \left|W_{1}\right|^{2} \leq\|\varphi\|_{L_{\tau}^{2} L_{\xi}^{2}}^{2} \\
& \times\left\|\frac{\langle\xi\rangle^{k}}{\left\langle\tau+\xi^{2}\right\rangle^{a}} \iint \frac{g\left(\xi_{1}, \tau_{1}\right) f\left(\xi-\xi_{1}, \tau-\tau_{1}\right) \chi_{\mathcal{R}_{1}} d \tau_{1} d \xi_{1}}{\left\langle\tau_{1}-\xi_{1}^{3}\right\rangle^{b}\left\langle\xi_{1}\right\rangle^{s}\left\langle\tau-\tau_{1}+\left(\xi-\xi_{1}\right)^{2}\right\rangle^{b}\left\langle\xi-\xi_{1}\right\rangle^{k}}\right\|_{L_{\tau}^{2} L_{\xi}^{2}}^{2} \\
& \leq \iint \frac{\langle\xi\rangle^{2 k}}{\left\langle\tau+\xi^{2}\right\rangle^{2 a}}\left|\iint \frac{g\left(\xi_{1}, \tau_{1}\right) f\left(\xi-\xi_{1}, \tau-\tau_{1}\right) \chi_{\mathcal{R}_{1}} d \tau_{1} d \xi_{1}}{\left\langle\tau_{1}-\xi_{1}^{3}\right\rangle^{b}\left\langle\xi_{1}\right\rangle^{s}\left\langle\tau-\tau_{1}+\left(\xi-\xi_{1}\right)^{2}\right\rangle^{b}\left\langle\xi-\xi_{1}\right\rangle^{k}}\right|^{2} d \tau d \xi \\
& \leq \iint \frac{\langle\xi\rangle^{2 k}}{\left\langle\tau+\xi^{2}\right\rangle^{2 a}}\left(\iint \frac{\chi_{\mathcal{R}_{1}} d \tau_{1} d \xi_{1}}{\left\langle\tau_{1}-\xi_{1}^{3}\right\rangle^{2 b}\left\langle\xi_{1}\right\rangle^{2 s}\left\langle\tau-\tau_{1}+\left(\xi-\xi_{1}\right)^{2}\right\rangle^{2 b}\left\langle\xi-\xi_{1}\right\rangle^{2 k}}\right. \\
& \left.\times \iint\left|g\left(\xi_{1}, \tau_{1}\right)\right|^{2}\left|f\left(\xi-\xi_{1}, \tau-\tau_{1}\right)\right|^{2} d \tau_{1} d \xi_{1}\right) d \tau d \xi \\
& \leq\|f\|_{L_{\tau}^{2} L_{\xi}^{2}}^{2}\|g\|_{L_{\tau_{1}}^{2} L_{\xi_{1}}^{2}}^{2} \\
& \times\left\|\frac{\langle\xi\rangle^{2 k}}{\left\langle\tau+\xi^{2}\right\rangle^{2 a}} \iint \frac{\chi_{\mathcal{R}_{1}} d \tau_{1} d \xi_{1}}{\left\langle\tau_{1}-\xi_{1}^{3}\right\rangle^{2 b}\left\langle\xi_{1}\right\rangle^{2 s}\left\langle\tau-\tau_{1}+\left(\xi-\xi_{1}\right)^{2}\right\rangle^{2 b}\left\langle\xi-\xi_{1}\right\rangle^{2 k}}\right\|_{L_{\tau}^{\infty} L_{\xi}^{\infty}} \\
& =\|u\|_{X^{k, b}}^{2}\|v\|_{Y^{s, b}}^{2} \\
& \times\left\|\frac{\langle\xi\rangle^{2 k}}{\left\langle\tau+\xi^{2}\right\rangle^{2 a}} \iint \frac{\chi_{\mathcal{R}_{1}} d \tau_{1} d \xi_{1}}{\left\langle\tau_{1}-\xi_{1}^{3}\right\rangle^{2 b}\left\langle\xi_{1}\right\rangle^{2 s}\left\langle\tau-\tau_{1}+\left(\xi-\xi_{1}\right)^{2}\right\rangle^{2 b}\left\langle\xi-\xi_{1}\right\rangle^{2 k}}\right\|_{L_{\tau}^{\infty} L_{\xi}^{\infty}} .
\end{aligned}
$$


For $W_{2}$ we put $\tilde{f}(\xi, \tau)=f(-\xi,-\tau)$, integrate over $\tau$ and $\xi$ first, and follow the same steps as above to get

$$
\begin{aligned}
& \left|W_{2}\right|^{2} \leq\|g\|_{L_{\tau_{1}}^{2} L_{\xi_{1}}^{2}}^{2} \\
& \quad \times\left\|\frac{1}{\left\langle\xi_{1}\right\rangle^{s}\left\langle\tau_{1}-\xi_{1}^{3}\right\rangle^{b}} \iint \frac{\langle\xi\rangle^{k} \widetilde{f}\left(\xi_{1}-\xi, \tau_{1}-\tau\right) \bar{\varphi}(\tau, \xi) \chi_{\mathcal{R}_{2}} d \tau d \xi}{\left\langle\tau+\xi^{2}\right\rangle^{a}\left\langle\tau-\tau_{1}+\left(\xi-\xi_{1}\right)^{2}\right\rangle^{b}\left\langle\xi-\xi_{1}\right\rangle^{k}}\right\|_{L_{\tau_{1}}^{2} L_{\xi_{1}}^{2}}^{2} \\
& \leq\|\widetilde{f}\|_{L_{\tau_{1}}^{2} L_{\xi_{1}}^{2}}^{2}\|g\|_{L_{\tau_{1}}^{2} L_{\xi_{1}}^{2}}^{2} \\
& \quad \times\left\|\frac{1}{\left\langle\xi_{1}\right\rangle^{2 s}\left\langle\tau_{1}-\xi_{1}^{3}\right\rangle^{2 b}} \iint \frac{\langle\xi\rangle^{2 k} \chi_{\mathcal{R}_{2}} d \tau d \xi}{\left\langle\tau+\xi^{2}\right\rangle^{2 a}\left\langle\tau-\tau_{1}+\left(\xi-\xi_{1}\right)^{2}\right\rangle^{2 b}\left\langle\xi-\xi_{1}\right\rangle^{2 k}}\right\|_{L_{\tau_{1}}^{\infty} L_{\xi_{1}}^{\infty}}^{2} \\
& =\|u\|_{X^{k, b}}^{2}\|v\|_{Y^{s, b}}^{2} \\
& \quad \times\left\|\frac{1}{\left\langle\xi_{1}\right\rangle^{2 s}\left\langle\tau_{1}-\xi_{1}^{3}\right\rangle^{2 b}} \iint \frac{\langle\xi\rangle^{2 k} \chi_{\mathcal{R}_{2}} d \tau d \xi}{\left\langle\tau+\xi^{2}\right\rangle^{2 a}\left\langle\tau-\tau_{1}+\left(\xi-\xi_{1}\right)^{2}\right\rangle^{2 b}\left\langle\xi-\xi_{1}\right\rangle^{2 k}}\right\|_{L_{\tau_{1}}^{\infty} L_{\xi_{1}}^{\infty}} .
\end{aligned}
$$

Note that $\widetilde{f}(\xi, \tau)=\langle\xi\rangle^{k}\left\langle\tau-\xi^{2}\right\rangle^{b} \widehat{u}(-\xi,-\tau)$ and $\|\widetilde{f}\|_{L_{\tau}^{2} L_{\xi}^{2}}=\|f\|_{L_{\tau}^{2} L_{\xi}^{2}}=\|u\|_{X^{k, b}}$.

Now using the change of variables $\tau=\tau_{1}-\tau_{2}$ and $\xi=\xi_{1}-\xi_{2}$ the third region, $\mathcal{R}_{3}$, is transformed into the set $\widetilde{\mathcal{R}}_{3}$ such that

$\widetilde{\mathcal{R}}_{3} \subseteq\left\{\left(\xi_{1}, \xi_{2}, \tau_{1}, \tau_{2}\right) \in \mathbb{R}^{4} ; \frac{1}{2}\left|\xi_{1}\right|^{3} \leq\left|\xi_{1}^{3}+\xi_{1}^{2}-2 \xi_{1} \xi_{2}\right| \leq 3\left|\tau_{2}-\xi_{2}^{2}\right| \quad\right.$ and $\left.\quad\left|\xi_{1}\right|>2\right\}$.

Then $W_{3}$ can be estimated as follows:

$$
\begin{aligned}
& \left|W_{3}\right|^{2} \leq\|\widetilde{f}\|_{L_{\tau_{2}}^{2} L_{\xi_{2}}^{2}}^{2} \\
& \quad \times\left\|\frac{1}{\left\langle\xi_{2}\right\rangle^{k}\left\langle\tau_{2}-\xi_{2}^{2}\right\rangle^{b}} \iint \frac{\left\langle\xi_{1}-\xi_{2}\right\rangle^{k} g\left(\xi_{1}, \tau_{1}\right) \tilde{\varphi}\left(\xi_{2}-\xi_{1}, \tau_{2}-\tau_{1}\right) \chi_{\widetilde{\mathcal{R}}_{3}} d \tau_{1} d \xi_{1}}{\left\langle\tau_{1}-\tau_{2}+\left(\xi_{1}-\xi_{2}\right)^{2}\right\rangle^{a}\left\langle\tau_{1}-\xi_{1}^{3}\right\rangle^{b}\left\langle\xi_{1}\right\rangle^{s}}\right\|_{L_{\tau_{2}}^{2} L_{\xi_{2}}^{2}}^{2} \\
& \leq\|\widetilde{f}\|_{L_{\tau_{2}}^{2} L_{\xi_{2}}^{2}}^{2}\|g\|_{L_{\tau_{1}}^{2} L_{\xi_{1}}^{2}}^{2} \\
& \quad \times\left\|\frac{1}{\left\langle\xi_{2}\right\rangle^{2 k}\left\langle\tau_{2}-\xi_{2}^{2}\right\rangle^{2 b}} \iint \frac{\left\langle\xi_{1}-\xi_{2}\right\rangle^{2 k} \chi_{\widetilde{\mathcal{R}}_{3}} d \tau_{1} d \xi_{1}}{\left\langle\tau_{1}-\tau_{2}+\left(\xi_{1}-\xi_{2}\right)^{2}\right\rangle^{2 a}\left\langle\tau_{1}-\xi_{1}^{3}\right\rangle^{2 b}\left\langle\xi_{1}\right\rangle^{2 s}}\right\|_{L_{\tau_{2}}^{\infty} L_{\xi_{2}}^{\infty}}^{2} \\
& =\|u\|_{X^{k, b}}^{2}\|v\|_{Y^{s, b}}^{2} \\
& \quad \times\left\|\frac{1}{\left\langle\xi_{2}\right\rangle^{2 k}\left\langle\tau_{2}-\xi_{2}^{2}\right\rangle^{2 b}} \iint \frac{\left\langle\xi_{1}-\xi_{2}\right\rangle^{2 k} \chi_{\widetilde{\mathcal{R}}_{3}} d \tau_{1} d \xi_{1}}{\left\langle\tau_{1}-\tau_{2}+\left(\xi_{1}-\xi_{2}\right)^{2}\right\rangle^{2 a}\left\langle\tau_{1}-\xi_{1}^{3}\right\rangle^{2 b}\left\langle\xi_{1}\right\rangle^{2 s}}\right\|_{L_{\tau_{2}}^{\infty} L_{\xi_{2}}^{\infty}} .
\end{aligned}
$$

From the estimates (3.7), (3.8) and (3.9) it suffices to show that the following expressions are bounded by a constant $C$ :

$$
\begin{aligned}
& \left\|\frac{\langle\xi\rangle^{2 k}}{\left\langle\tau+\xi^{2}\right\rangle^{2 a}} \iint \frac{\chi_{\mathcal{R}_{1}} d \tau_{1} d \xi_{1}}{\left\langle\tau_{1}-\xi_{1}^{3}\right\rangle^{2 b}\left\langle\xi_{1}\right\rangle^{2 s}\left\langle\tau-\tau_{1}+\left(\xi-\xi_{1}\right)^{2}\right\rangle^{2 b}\left\langle\xi-\xi_{1}\right\rangle^{2 k}}\right\|_{L_{\tau}^{\infty} L_{\xi}^{\infty}}, \\
& \left\|\frac{1}{\left\langle\xi_{1}\right\rangle^{2 s}\left\langle\tau_{1}-\xi_{1}^{3}\right\rangle^{2 b}} \iint \frac{\langle\xi\rangle^{2 k} \chi_{\mathcal{R}_{2}} d \tau d \xi}{\left\langle\tau+\xi^{2}\right\rangle^{2 a}\left\langle\tau-\tau_{1}+\left(\xi-\xi_{1}\right)^{2}\right\rangle^{2 b}\left\langle\xi-\xi_{1}\right\rangle^{2 k}}\right\|_{L_{\tau_{1}}^{\infty} L_{\xi_{1}}^{\infty}}, \\
& \left\|\frac{1}{\left\langle\xi_{2}\right\rangle^{2 k}\left\langle\tau_{2}-\xi_{2}^{2}\right\rangle^{2 b}} \iint \frac{\left\langle\xi_{1}-\xi_{2}\right\rangle^{2 k} \chi_{\widetilde{\mathcal{R}}_{3}} d \tau_{1} d \xi_{1}}{\left\langle\tau_{1}-\tau_{2}+\left(\xi_{1}-\xi_{2}\right)^{2}\right\rangle^{2 a}\left\langle\tau_{1}-\xi_{1}^{3}\right\rangle^{2 b}\left\langle\xi_{1}\right\rangle^{2 s}}\right\|_{L_{\tau_{2}}^{\infty} L_{\xi_{2}}^{\infty}},
\end{aligned}
$$


According to (2.11) with $\mu=1$ in Lemma 2.4, noting that $\langle\xi\rangle \leq\left\langle\xi_{1}\right\rangle\left\langle\xi-\xi_{1}\right\rangle$ and $\left\langle\xi_{1}-\xi_{2}\right\rangle \leq\left\langle\xi_{1}\right\rangle\left\langle\xi_{2}\right\rangle$ and using that $k \geq 0$ it suffices to get bounds for

$$
\begin{gathered}
\widetilde{W}_{1}(\tau, \xi)=\frac{1}{\left\langle\tau+\xi^{2}\right\rangle^{2 a}} \int \frac{\left\langle\xi_{1}\right\rangle^{2 k-2 s} d \xi_{1}}{\left\langle\tau+\xi^{2}-\xi_{1}^{3}+\xi_{1}^{2}-2 \xi \xi_{1}\right\rangle^{2 b}} \quad \text { on } \quad \mathcal{R}_{1}, \\
\widetilde{W}_{2}\left(\tau_{1}, \xi_{1}\right)=\frac{\left\langle\xi_{1}\right\rangle^{2 k-2 s}}{\left\langle\tau_{1}-\xi_{1}^{3}\right\rangle^{2 b}} \int \frac{d \xi}{\left\langle\tau_{1}-\xi_{1}^{2}+2 \xi \xi_{1}\right\rangle^{2 a}} \quad \text { on } \quad \mathcal{R}_{2}, \\
\widetilde{W}_{3}\left(\tau_{2}, \xi_{2}\right)=\frac{1}{\left\langle\tau_{2}-\xi_{2}^{2}\right\rangle^{2 b}} \int \frac{\left\langle\xi_{1}\right\rangle^{2 k-2 s} d \xi_{1}}{\left\langle\tau_{2}-\xi_{2}^{2}-\xi_{1}^{3}-\xi_{1}^{2}+2 \xi_{1} \xi_{2}\right\rangle^{2 a}} \quad \text { on } \quad \widetilde{\mathcal{R}}_{3},
\end{gathered}
$$

where we have used that $\min \{a, b\}=a$ and $b>\frac{1}{2}$.

We begin by estimating $\widetilde{W}_{1}$ on $\mathcal{R}_{1}=\mathcal{A} \cup \mathcal{B} \cup \mathcal{C}_{1}$. In region $\mathcal{A}$, using $\left|\xi_{1}\right| \leq 2, a>0$ and $b>\frac{1}{2}$ we see that

$$
\left|\widetilde{W}_{1}(\tau, \xi)\right| \leq C \int \frac{d \xi_{1}}{\left\langle\tau+\xi^{2}-\xi_{1}^{3}+\xi_{1}^{2}-2 \xi \xi_{1}\right\rangle^{2 b}} \leq C .
$$

In region $\mathcal{B}$, by the change of variables $\eta=\tau+\xi^{2}-\xi_{1}^{3}+\xi_{1}^{2}-2 \xi \xi_{1}$ and the condition $\left|3 \xi_{1}^{2}-2 \xi_{1}+2 \xi\right| \geq \frac{1}{2}\left|\xi_{1}\right|^{2}$ we obtain

$$
\begin{aligned}
\left|\widetilde{W}_{1}(\tau, \xi)\right| & \leq \frac{1}{\left\langle\tau+\xi^{2}\right\rangle^{2 a}} \int \frac{\left\langle\xi_{1}\right\rangle^{2 k-2 s}}{\left|3 \xi_{1}^{2}-2 \xi_{1}+2 \xi\right|\langle\eta\rangle^{2 b}} d \eta \\
& \leq \frac{1}{\left\langle\tau+\xi^{2}\right\rangle^{2 a}} \int \frac{\left\langle\xi_{1}\right\rangle^{2 k-2 s}}{\left|\xi_{1}\right|^{2}\langle\eta\rangle^{2 b}} d \eta \leq C .
\end{aligned}
$$

Here we have used $a>0, k-s \leq \min \{1,3 a\},\left|\xi_{1}\right|>2$ and (2.12) in Lemma 2.4.

In region $\mathcal{C}_{1}$, by (3.6) we have that

$$
\frac{1}{2}\left|\xi_{1}\right|^{3} \leq 3\left|\tau+\xi^{2}\right|<3\left\langle\tau+\xi^{2}\right\rangle
$$

and consequently using $a>0$ we obtain

$$
\left\langle\tau+\xi^{2}\right\rangle^{-2 a} \leq C\left|\xi_{1}\right|^{-6 a} .
$$

Then use $k-s \leq \min \{1,3 a\}$ combined with (2.13) of Lemma 2.4 to get

$$
\begin{aligned}
\left|\widetilde{W}_{1}(\tau, \xi)\right| & \leq \int \frac{\left\langle\xi_{1}\right\rangle^{2 k-2 s}}{\left\langle\tau+\xi^{2}\right\rangle^{2 a}\left\langle\tau+\xi^{2}-\xi_{1}^{3}+\xi_{1}^{2}-2 \xi \xi_{1}\right\rangle^{2 b}} d \xi_{1} \\
& \leq C \int \frac{\left\langle\xi_{1}\right\rangle^{2 k-2 s}}{\left|\xi_{1}\right|^{6 a}\left\langle\tau+\xi^{2}-\xi_{1}^{3}+\xi_{1}^{2}-2 \xi \xi_{1}\right\rangle^{2 b}} d \xi_{1} \\
& \leq C .
\end{aligned}
$$

Next we estimate $\widetilde{W}_{2}$. Making the change of variables, $\eta=\tau_{1}-\xi_{1}^{2}+2 \xi \xi_{1}$, using (3.6) and the restriction in region $\mathcal{C}_{2}$, we have

$$
|\eta| \leq\left|\tau_{1}-\xi_{1}^{3}\right|+\left|\xi_{1}^{3}-\xi_{1}^{2}+2 \xi \xi_{1}\right| \leq 4\left|\tau_{1}-\xi_{1}^{3}\right| \leq 4\left\langle\tau_{1}-\xi_{1}^{3}\right\rangle .
$$

Moreover in $\mathcal{C}_{2}$, since $a+b-\frac{1}{2}>0$, we have

$$
\frac{1}{2}\left|\xi_{1}\right|^{3} \leq 3\left|\tau_{1}-\xi_{1}^{3}\right|<3\left\langle\tau_{1}-\xi_{1}^{3}\right\rangle
$$

and hence

$$
\left\langle\tau_{1}-\xi_{1}^{3}\right\rangle^{-(2 a+2 b-1)} \leq C\left|\xi_{1}\right|^{-3(2 a+2 b-1)} .
$$


Then we can estimate $\widetilde{W}_{2}$ as follows:

$$
\begin{aligned}
\left|\widetilde{W}_{2}\left(\tau_{1}, \xi_{1}\right)\right| & \leq \frac{\left\langle\xi_{1}\right\rangle^{2 k-2 s}}{\left\langle\tau_{1}-\xi_{1}^{3}\right\rangle^{2 b}} \int_{|\eta| \leq 4\left\langle\tau_{1}-\xi_{1}^{3}\right\rangle} \frac{(1+|\eta|)^{-2 a}}{2\left|\xi_{1}\right|} d \eta \\
& =\frac{\left\langle\xi_{1}\right\rangle^{2 k-2 s}}{\left\langle\tau_{1}-\xi_{1}^{3}\right\rangle^{2 b}\left|\xi_{1}\right|} \frac{1}{(1-2 a)}\left(\left(4\left\langle\tau_{1}-\xi_{1}^{3}\right\rangle\right)^{1-2 a}-1\right) \\
& \leq C \frac{\left\langle\xi_{1}\right\rangle^{2 k-2 s}}{\left|\xi_{1}\right|\left\langle\tau_{1}-\xi_{1}^{3}\right\rangle^{2 a+2 b-1}} \\
& \leq C \frac{\left\langle\xi_{1}\right\rangle^{2 k-2 s}}{\left|\xi_{1}\right|^{6 a+6 b-2}} \leq C,
\end{aligned}
$$

where in the last inequality we have used that $k-s \leq 3 a+3 b-1$, which follows from the conditions $k-s \leq \min \{1,3 a\}$ and $b>\frac{1}{2}$.

Finally, in the region $\widetilde{\mathcal{R}}_{3}$ we note that

$$
\frac{1}{2}\left|\xi_{1}\right|^{3}<3\left\langle\tau_{2}-\xi_{2}^{2}\right\rangle \Longrightarrow\left\langle\tau_{2}-\xi_{2}^{2}\right\rangle^{-2 b} \leq C\left|\xi_{1}\right|^{-6 b}
$$

and from the conditions $k-s \leq \min \{1,3 a\}$ and $\frac{1}{6}<a<\frac{1}{2}<b$ coupled with (2.13) of Lemma 2.4, we have that

$$
\left|\widetilde{W}_{3}\left(\tau_{2}, \xi_{2}\right)\right| \leq C \int \frac{\left\langle\xi_{1}\right\rangle^{2 k-2 s} d \xi_{1}}{\left|\xi_{1}\right|^{6 b}\left\langle\tau_{2}-\xi_{2}^{2}-\xi_{1}^{3}-\xi_{1}^{2}+2 \xi_{1} \xi_{2}\right\rangle^{2 a}} \leq C,
$$

and hence the proof of Lemma 3.1 is completed.

\subsection{Proof of Lemma 3.2. We let}

$$
\left\{\begin{array}{l}
\tau=\tau_{1}-\tau_{2}, \quad \xi=\xi_{1}-\xi_{2} \\
\sigma=\tau-\xi^{3}, \quad \sigma_{1}=\tau_{1}+\xi_{1}^{2}, \quad \sigma_{2}=\tau_{2}+\xi_{2}^{2},
\end{array}\right.
$$

and define

$$
f\left(\xi_{1}, \tau_{1}\right)=\left\langle\xi_{1}\right\rangle^{k}\left\langle\sigma_{1}\right\rangle^{b} \widehat{u}_{1}\left(\xi_{1}, \tau_{1}\right) \quad \text { and } \quad g\left(\xi_{2}, \tau_{2}\right)=\left\langle\xi_{2}\right\rangle^{k}\left\langle\sigma_{2}\right\rangle^{b} \widehat{\bar{u}}_{2}\left(-\xi_{2},-\tau_{2}\right) .
$$

Hence we have

$$
\left\|u_{1}\right\|_{X^{k, b}}=\|f\|_{L_{\tau_{1}}^{2} L_{\xi_{1}}^{2}} \quad \text { and } \quad\left\|u_{2}\right\|_{X^{k, b}}=\|g\|_{L_{\tau_{2}}^{2} L_{\xi_{2}}^{2}} .
$$

Now using (3.21), (3.22) and (3.23) we estimate the left-hand side of (3.3) as in the proof of Lemma 3.1 to obtain

$$
\begin{aligned}
\left\|\partial_{x}\left(u_{1} \bar{u}_{2}\right)\right\|_{Y^{s,-a}} & =\left\|\langle\sigma\rangle^{-a}\langle\xi\rangle^{s} \partial_{x}{\widehat{\left(u_{1} \bar{u}_{2}\right.}}_{2}\right\|_{L_{\tau}^{2} L_{\xi}^{2}} \\
& =\left\|i \xi\langle\sigma\rangle^{-a}\langle\xi\rangle^{s} \widehat{u}_{1} * \widehat{\bar{u}}_{2}(\tau, \xi)\right\|_{L_{\tau}^{2} L_{\xi}^{2}} \\
& =\sup _{\|\varphi\|_{L_{\tau, \xi}^{2}} \leq 1}\left|I\left(\frac{|\xi|\langle\xi\rangle^{s}}{\langle\sigma\rangle^{a}}{\widehat{u_{1}}} * \widehat{\bar{u}}_{2}, \varphi\right)\right| \\
& :=\sup _{\|\varphi\|_{L_{\tau, \xi}^{2}} \leq 1}\left|Z\left(u_{1}, u_{2}, \varphi\right)\right|,
\end{aligned}
$$

where

$$
\begin{aligned}
Z\left(u_{1}, u_{2}, \varphi\right) & =\iiint \int_{\mathbb{R}^{4}} \frac{|\xi|\langle\xi\rangle^{s}}{\langle\sigma\rangle^{a}} \widehat{u}_{1}\left(\xi_{1}, \tau_{1}\right) \widehat{\bar{u}}_{2}\left(\xi-\xi_{1}, \tau-\tau_{1}\right) \bar{\varphi}(\xi, \tau) d \tau_{1} d \xi_{1} d \tau d \xi \\
& =\iiint \int_{\mathbb{R}^{4}} \frac{|\xi|\langle\xi\rangle^{s} f\left(\xi_{1}, \tau_{1}\right) g\left(\xi_{2}, \tau_{2}\right) \bar{\varphi}(\xi, \tau)}{\langle\sigma\rangle^{a}\left\langle\xi_{1}\right\rangle^{k}\left\langle\sigma_{1}\right\rangle^{b}\left\langle\xi_{2}\right\rangle^{k}\left\langle\sigma_{2}\right\rangle^{b}} d \tau_{1} d \xi_{1} d \tau d \xi
\end{aligned}
$$


and hence by applying the Cauchy-Schwarz inequality, we have that

$$
\begin{aligned}
& \left|Z\left(u_{1}, u_{2}, \varphi\right)\right|^{2} \leq\|\varphi\|_{L_{\tau}^{2} L_{\xi}^{2}}^{2} \\
& \quad \times\left\|\frac{|\xi|\langle\xi\rangle^{s}}{\langle\sigma\rangle^{a}} \iint \frac{f\left(\xi+\xi_{2}, \tau+\tau_{2}\right) g\left(\xi_{2}, \tau_{2}\right)}{\left\langle\xi_{1}\right\rangle^{k}\left\langle\sigma_{1}\right\rangle^{b}\left\langle\xi_{2}\right\rangle^{k}\left\langle\sigma_{2}\right\rangle^{b}} d \tau_{2} d \xi_{2}\right\|_{L_{\tau}^{2} L_{\xi}^{2}} \\
& \leq\|f\|_{L_{\tau}^{2} L_{\xi}^{2}}^{2}\|g\|_{L_{\tau_{2}}^{2} L_{\xi_{2}}^{2}}^{2}\left\|\frac{|\xi|^{2}\langle\xi\rangle^{2 s}}{\langle\sigma\rangle^{2 a}} \iint \frac{d \tau_{2} d \xi_{2}}{\left\langle\xi_{1}\right\rangle^{2 k}\left\langle\sigma_{1}\right\rangle^{2 b}\left\langle\xi_{2}\right\rangle^{2 k}\left\langle\sigma_{2}\right\rangle^{2 b}}\right\|_{L_{\tau}^{\infty} L_{\xi}^{\infty}} \\
& =\left\|u_{1}\right\|_{X^{k, b}}^{2}\left\|u_{2}\right\|_{X^{k, b}}^{2}\left\|\frac{|\xi|^{2}\langle\xi\rangle^{2 s}}{\langle\sigma\rangle^{2 a}} \iint \frac{d \tau_{2} d \xi_{2}}{\left\langle\xi_{1}\right\rangle^{2 k}\left\langle\sigma_{1}\right\rangle^{2 b}\left\langle\xi_{2}\right\rangle^{2 k}\left\langle\sigma_{2}\right\rangle^{2 b}}\right\|_{L_{\tau}^{\infty} L_{\xi}^{\infty}} .
\end{aligned}
$$

Then it suffices to get bounds for

$$
\widetilde{Z}(\tau, \xi):=\frac{|\xi|^{2}\langle\xi\rangle^{2 s}}{\langle\sigma\rangle^{2 a}} \iint \frac{d \tau_{2} d \xi_{2}}{\left\langle\xi_{1}\right\rangle^{2 k}\left\langle\sigma_{1}\right\rangle^{2 b}\left\langle\xi_{2}\right\rangle^{2 k}\left\langle\sigma_{2}\right\rangle^{2 b}} .
$$

In order to estimate $\widetilde{Z}$ we consider several cases.

Case A. $|\boldsymbol{\xi}|<\mathbf{2}$. Using $k \geq 0, b>\frac{1}{2}, a \geq 0$, (2.11) of Lemma 2.4 with $\mu=1$ and (2.12) of Lemma 2.4, we have

$$
\begin{aligned}
\widetilde{Z}(\tau, \xi) & \leq \frac{|\xi|^{2}\langle\xi\rangle^{2 s}}{\langle\sigma\rangle^{2 a}} \int d \xi_{2} \int \frac{d \tau_{2}}{\left\langle\tau_{2}+\tau+\left(\xi_{2}+\xi\right)^{2}\right\rangle^{2 b}\left\langle\tau_{2}+\xi_{2}^{2}\right\rangle^{2 b}} \\
& \leq C \frac{|\xi|^{2}\langle\xi\rangle^{2 s}}{\langle\sigma\rangle^{2 a}} \int \frac{d \xi_{2}}{\left\langle\tau+\xi^{2}+2 \xi \xi_{2}\right\rangle^{2 b}} \\
& \leq C \frac{|\xi|^{2}\langle\xi\rangle^{2 s}}{\langle\sigma\rangle^{2 a}} \frac{1}{|\xi|} \leq C|\xi|\langle\xi\rangle^{2 s} \leq C .
\end{aligned}
$$

Case B. $|\xi| \geq 2$ and $\left|\xi_{1}\right| \geq 2\left|\xi_{2}\right|$. In this situation we have

$|\xi| \geq 2 \Longrightarrow|\xi|^{2} \leq \frac{1}{2}|\xi|^{3} \Longrightarrow\left|\xi^{3} \pm \xi^{2}\right| \geq|\xi|^{3}-|\xi|^{2} \geq \frac{1}{2}|\xi|^{3} \Longrightarrow\left\langle\xi^{3} \pm \xi^{2}\right\rangle \geq \frac{1}{2}\left\langle\xi^{3}\right\rangle$,

$$
\begin{gathered}
\left|\xi_{1}\right| \geq 2\left|\xi_{2}\right| \Longrightarrow|\xi| \leq\left|\xi_{1}\right|+\left|\xi_{2}\right| \leq \frac{3}{2}\left|\xi_{1}\right| \Longrightarrow\langle\xi\rangle^{2 k} \leq C\left\langle\xi_{1}\right\rangle^{2 k} \\
\left|\xi_{1}\right| \geq 2\left|\xi_{2}\right| \Longrightarrow|\xi| \geq\left|\xi_{1}\right|-\left|\xi_{2}\right| \geq\left|\xi_{2}\right|
\end{gathered}
$$

Now we consider two situations for parameters $k$ and $b$.

Case B-1. $\boldsymbol{k}>\frac{1}{2}$ and $\boldsymbol{b} \in\left(\frac{1}{2}, \boldsymbol{k}\right]$. Apply (2.11) with $\mu=1$ in Lemma 2.4 and (3.28) to get

$$
\begin{aligned}
\widetilde{Z}(\tau, \xi) & \leq C \frac{\langle\xi\rangle^{2 s}|\xi|^{2}\langle\xi\rangle^{-2 k}}{\langle\sigma\rangle^{2 a}} \int \frac{d \xi_{2}}{\left\langle\xi_{2}\right\rangle^{2 k}} \int \frac{d \tau_{2}}{\left\langle\tau+\tau_{2}+\left(\xi+\xi_{2}\right)^{2}\right\rangle^{2 b}\left\langle\tau_{2}+\xi_{2}^{2}\right\rangle^{2 b}} \\
& \leq C \frac{\langle\xi\rangle^{2 s-2 k+2}}{\langle\sigma\rangle^{2 a}} \int \frac{d \xi_{2}}{\left\langle\xi_{2}\right\rangle^{2 k}\left\langle\tau+\xi^{2}+2 \xi \xi_{2}\right\rangle^{2 b}} \\
& :=C \frac{\langle\xi\rangle^{2 s-2 k+2}}{\langle\sigma\rangle^{2 a}} \widetilde{Z}_{1}(\tau, \xi) .
\end{aligned}
$$


Now using $\frac{1}{2}<b \leq k$, (2.11) of Lemma 2.4 with $\mu(\xi)=\frac{1}{2|\xi|}$ and (3.31) we compute $\widetilde{Z}_{1}$ in the following way:

$$
\begin{aligned}
\widetilde{Z}_{1}(\tau, \xi) & =\int \frac{d \xi_{2}}{\left(1+\left|\tau+\xi^{2}+2 \xi \xi_{2}\right|\right)^{2 b}\left(1+\left|\xi_{2}\right|\right)^{2 k}} \\
& \leq \int \frac{d \xi_{2}}{\left(1+\left|\tau+\xi^{2}+2 \xi \xi_{2}\right|\right)^{2 b}\left(1+\left|\xi_{2}\right|\right)^{2 b}} \\
& =(2|\xi|)^{-2 b} \int \frac{d \xi_{2}}{\left(\frac{1}{2|\xi|}+\left|\xi_{2}+\frac{1}{2 \xi}\left(\tau+\xi^{2}\right)\right|\right)^{2 b}\left(1+\left|\xi_{2}\right|\right)^{2 b}} \\
& \leq(2|\xi|)^{-2 b} \int \frac{d \xi_{2}}{\left(\frac{1}{2|\xi|}+\left|\xi_{2}+\frac{1}{2 \xi}\left(\tau+\xi^{2}\right)\right|\right)^{2 b}\left(\frac{1}{2|\xi|}+\left|\xi_{2}\right|\right)^{2 b}} \\
& \leq C(2|\xi|)^{-2 b} \frac{(2|\xi|)^{-(1-2 b)}}{\left(\frac{1}{2|\xi|}+\frac{1}{2|\xi|}\left|\tau+\xi^{2}\right|\right)^{2 b}} \\
& =C \frac{(2|\xi|)^{2 b-1}}{\left(1+\left|\tau+\xi^{2}\right|\right)^{2 b}}=C \frac{(2|\xi|)^{2 b-1}}{\left\langle\tau+\xi^{2}\right\rangle^{2 b}} .
\end{aligned}
$$

Combining (3.32) and (3.33) and using $0 \leq a \leq b,\left\langle\xi^{3}+\xi^{2}\right\rangle \leq\left\langle\tau-\xi^{3}\right\rangle\left\langle\tau+\xi^{2}\right\rangle$ and (3.30) we have

$$
\begin{aligned}
\widetilde{Z}(\tau, \xi) & \leq C \frac{\langle\xi\rangle^{2 s-2 k+2}(2|\xi|)^{2 b-1}}{\langle\sigma\rangle^{2 a}\left\langle\tau+\xi^{2}\right\rangle^{2 b}} \leq C \frac{\langle\xi\rangle^{2 s-2 k+2 b+1}}{\left\langle\tau-\xi^{3}\right\rangle^{2 a}\left\langle\tau+\xi^{2}\right\rangle^{2 a}} \\
& \leq C \frac{\langle\xi\rangle^{2 s-2 k+2 b+1}}{\left\langle\xi^{3}+\xi^{2}\right\rangle^{2 a}} \leq C \frac{\langle\xi\rangle^{2 s-2 k+2 b+1}}{\left\langle\xi^{3}\right\rangle^{2 a}} \\
& \leq C \frac{\langle\xi\rangle^{2 s-2 k+2 b+1}}{\langle\xi\rangle^{6 a}} \leq C,
\end{aligned}
$$

where it was used in the inequality $s-k+b+\frac{1}{2} \leq 3 a$ which holds from hypothesis (ii).

Case B-2. $k \in\left[\mathbf{0}, \frac{1}{2}\right]$ and $\boldsymbol{b}>\frac{1}{2}$. We note that in the previous situation to estimate $\widetilde{Z}_{1}$ we obtained the following estimate:

$$
\int \frac{d \xi_{2}}{\left(1+\left|\tau+\xi^{2}+2 \xi \xi_{2}\right|\right)^{2 b}\left(1+\left|\xi_{2}\right|\right)^{2 b}} \leq C \frac{(2|\xi|)^{2 b-1}}{\left\langle\tau+\xi^{2}\right\rangle^{2 b}} .
$$

On the other hand using $0 \leq k \leq \frac{1}{2}, b>\frac{1}{2}$ and (3.29) we have

$$
\left\langle\xi_{2}\right\rangle^{2 b-2 k} \leq\langle\xi\rangle^{2 b-2 k} \text {. }
$$

Then by (3.35), (3.36) and (3.30) we have

$$
\begin{aligned}
\widetilde{Z}(\tau, \xi) & \leq C \frac{\langle\xi\rangle^{2 s-2 k+2}}{\langle\sigma\rangle^{2 a}} \int \frac{d \xi_{2}}{\left\langle\xi_{2}\right\rangle^{2 k}\left\langle\tau+\xi^{2}+2 \xi \xi_{2}\right\rangle^{2 b}} \\
& =C \frac{\langle\xi\rangle^{2 s-2 k+2}}{\langle\sigma\rangle^{2 a}} \int \frac{\left\langle\xi_{2}\right\rangle^{2 b-2 k} d \xi_{2}}{\left(1+\left|\tau+\xi^{2}+2 \xi \xi_{2}\right|\right)^{2 b}\left(1+\left|\xi_{2}\right|\right)^{2 b}} \\
& \leq C \frac{\langle\xi\rangle^{2 s-4 k+2 b+2}}{\left\langle\tau-\xi^{3}\right\rangle^{2 a}} \frac{(2|\xi|)^{2 b-1}}{\left\langle\tau+\xi^{2}\right\rangle^{2 b}} \\
& \leq C \frac{\langle\xi\rangle^{2 s-4 k+4 b+1}}{\left\langle\xi^{3}\right\rangle^{2 a}} \leq C \frac{\langle\xi\rangle^{2 s-4 k+4 b+1}}{\langle\xi\rangle^{6 a}} \leq C,
\end{aligned}
$$


where it was used in the inequality $s-2 k+2 b+\frac{1}{2} \leq 3 a$ which holds from hypothesis (i).

Case C. $|\boldsymbol{\xi}| \geq \mathbf{2}$ and $\left|\boldsymbol{\xi}_{2}\right| \geq \mathbf{2}\left|\boldsymbol{\xi}_{\mathbf{1}}\right|$. Clearly, we have (3.30) and (3.31). If we interchange the positions of $\xi_{1}$ and $\xi_{2}$ in (3.28), (3.29) we get

$$
\begin{gathered}
\langle\xi\rangle^{2 k} \leq C\left\langle\xi_{2}\right\rangle^{2 k}, \\
|\xi| \geq\left|\xi_{1}\right| .
\end{gathered}
$$

Now using $\xi_{2}=\xi_{1}-\xi$ and $\tau_{2}=\tau_{1}-\tau$ we have

$$
\iint \frac{d \xi_{2} d \tau_{2}}{\left\langle\xi_{1}\right\rangle^{2 k}\left\langle\sigma_{1}\right\rangle^{2 b}\left\langle\xi_{2}\right\rangle^{2 k}\left\langle\sigma_{2}\right\rangle^{2 b}}=\iint \frac{d \xi_{1} d \tau_{1}}{\left\langle\xi_{1}\right\rangle^{2 k}\left\langle\sigma_{1}\right\rangle^{2 b}\left\langle\xi_{2}\right\rangle^{2 k}\left\langle\sigma_{2}\right\rangle^{2 b}} .
$$

Similar to case B we consider two situations for parameters $k$ and $b$.

Case C-1. $\boldsymbol{k}>\frac{1}{2}$ and $\boldsymbol{b} \in\left(\frac{1}{2}, \boldsymbol{k}\right]$. Again we apply (2.11) with $\mu=1$ in Lemma 2.4, (3.38) and (3.40) to get

$$
\begin{aligned}
\widetilde{Z}(\tau, \xi) & \leq C \frac{\langle\xi\rangle^{2 s}|\xi|^{2}\langle\xi\rangle^{-2 k}}{\langle\sigma\rangle^{2 a}} \int \frac{d \xi_{1}}{\left\langle\xi_{1}\right\rangle^{2 k}} \int \frac{d \tau_{1}}{\left\langle\tau_{1}-\tau+\left(\xi_{1}-\xi\right)^{2}\right\rangle^{2 b}\left\langle\tau_{1}+\xi_{1}^{2}\right\rangle^{2 b}} \\
& \leq C \frac{\langle\xi\rangle^{2 s-2 k+2}}{\langle\sigma\rangle^{2 a}} \int \frac{d \xi_{1}}{\left\langle\xi_{1}\right\rangle^{2 k}\left\langle\tau-\xi^{2}+2 \xi \xi_{1}\right\rangle^{2 b}} \\
& :=C \frac{\langle\xi\rangle^{2 s-2 k+2}}{\langle\sigma\rangle^{2 a}} \widetilde{Z}_{2}(\tau, \xi) .
\end{aligned}
$$

Now using $\frac{1}{2}<b \leq k$, (2.11) of Lemma 2.4 with $\mu(\xi)=\frac{1}{2|\xi|}$ and (3.31) we estimate $\widetilde{Z}_{2}(\tau, \xi)$ similar to $\widetilde{Z}_{1}(\tau, \xi)$ in (3.33) to get

$$
\widetilde{Z}_{2}(\tau, \xi) \leq \frac{C(2|\xi|)^{2 b-1}}{\left\langle\tau-\xi^{2}\right\rangle^{2 b}} .
$$

Combining (3.41) and (3.42) and using $0 \leq a \leq b,\left\langle\xi^{3}-\xi^{2}\right\rangle \leq\left\langle\tau-\xi^{3}\right\rangle\left\langle\tau-\xi^{2}\right\rangle$ and (3.30) we have

$$
\begin{aligned}
\widetilde{Z}(\tau, \xi) & \leq C \frac{\langle\xi\rangle^{2 s-2 k+2}(2|\xi|)^{2 b-1}}{\langle\sigma\rangle^{2 a}\left\langle\tau-\xi^{2}\right\rangle^{2 b}} \leq C \frac{\langle\xi\rangle^{2 s-2 k+2 b+1}}{\left\langle\tau-\xi^{3}\right\rangle^{2 a}\left\langle\tau-\xi^{2}\right\rangle^{2 a}} \\
& \leq C \frac{\langle\xi\rangle^{2 s-2 k+2 b+1}}{\left\langle\xi^{3}-\xi^{2}\right\rangle^{2 a}} \leq C \frac{\langle\xi\rangle^{2 s-2 k+2 b+1}}{\left\langle\xi^{3}\right\rangle^{2 a}} \\
& \leq C \frac{\langle\xi\rangle^{2 s-2 k+2 b+1}}{\langle\xi\rangle^{6 a}} \leq C,
\end{aligned}
$$

where we have used the inequality $s-k+b+\frac{1}{2} \leq 3 a$ which holds from hypothesis (ii).

Case C-2. $\boldsymbol{k} \in\left[\mathbf{0}, \frac{\mathbf{1}}{2}\right]$ and $\boldsymbol{b}>\mathbf{1 / 2}$. Here the calculations are the same as in Case B-2.

Case D. $|\xi| \geq 2$ and $\frac{1}{2}\left|\xi_{2}\right| \leq\left|\xi_{1}\right| \leq 2\left|\xi_{2}\right|$. Now we have

$$
|\xi|=\left|\xi_{1}-\xi_{2}\right| \leq\left|\xi_{1}\right|+\left|\xi_{2}\right| \leq \min \left\{3\left|\xi_{1}\right|, 3\left|\xi_{2}\right|\right\}
$$

and hence $k \geq 0$ implies

$$
\left\langle\xi_{1}\right\rangle^{-2 k}\left\langle\xi_{2}\right\rangle^{-2 k} \leq C\langle\xi\rangle^{-4 k}
$$


Then (2.11) of Lemma 2.4 with $\mu=1$, (3.44) and $a \geq 0$ gives

$$
\begin{aligned}
\widetilde{Z}(\tau, \xi) & =\frac{|\xi|^{2}\langle\xi\rangle^{2 s}}{\langle\sigma\rangle^{2 a}} \int d \xi_{2} \int \frac{d \tau_{2}}{\left\langle\xi_{1}\right\rangle^{2 k}\left\langle\xi_{2}\right\rangle^{2 k}\left\langle\tau_{2}+\tau+\left(\xi_{2}+\xi\right)^{2}\right\rangle^{2 b}\left\langle\tau_{2}+\xi_{2}^{2}\right\rangle^{2 b}} \\
& \leq C \frac{\langle\xi\rangle^{2 s-4 k}|\xi|^{2}}{\langle\sigma\rangle^{2 a}} \int \frac{d \xi_{2}}{\left(1+\left|\tau+\xi^{2}+\xi \xi_{2}\right|^{2}\right)^{2 b}} \\
& \leq C \frac{\langle\xi\rangle^{2 s-4 k}|\xi|^{2}}{\langle\sigma\rangle^{2 a} 2|\xi|} \leq C \frac{\langle\xi\rangle^{2 s-4 k+1}}{\left\langle\tau-\xi^{3}\right\rangle^{2 a}} \\
& \leq C\langle\xi\rangle^{2 s-4 k+1} \leq C
\end{aligned}
$$

where we have used the inequality $s-2 k+\frac{1}{2} \leq 0$.

We note that (3.30) and (3.31) hold in this case. Moreover $|\xi| \leq\left|\xi_{1}\right|+\left|\xi_{2}\right| \leq$ $3\left|\xi_{1}\right|$ and $k \geq 0$ imply $\langle\xi\rangle^{2 k} \leq C\left\langle\xi_{1}\right\rangle^{2 k}$ as in (3.28). Consequently, if $k>\frac{1}{2}$ and $b \in\left[\frac{1}{2}, k\right]$ we can compute $\widetilde{Z}(\tau, \xi)$ similar to Case B-1 to obtain $\widetilde{Z}(\tau, \xi) \leq C$ for $s-k+b+\frac{1}{2} \leq 3 a$.

Collecting the estimates given in the above cases, we complete the proof of Lemma 3.2

\section{LOCAL EXISTENCE FOR LOW REGULARITY DATA}

In this section we prove Theorem 1.1 regarding local well-posedness for the IVP (1.1).

The Cauchy problem (1.1) is rewritten in a standard way as the integral system

$$
\begin{aligned}
& u(t)=U(t) u_{0}-i \int_{0}^{t} U\left(t-t^{\prime}\right)\left\{\alpha(u v)\left(t^{\prime}\right)+\beta\left(|u|^{2} u\right)\left(t^{\prime}\right)\right\} d t^{\prime}, \\
& v(t)=V(t) v_{0}+\int_{0}^{t} V\left(t-t^{\prime}\right)\left\{\gamma \partial_{x}\left(|u|^{2}\right)\left(t^{\prime}\right)-\frac{1}{2} \partial_{x}\left(v^{2}\right)\left(t^{\prime}\right)\right\} d t^{\prime} .
\end{aligned}
$$

One replaces the system (4.1) by the cut-off system

$$
\begin{aligned}
& u(t)=\psi_{1}(t) U(t) u_{0}-i \psi_{T}(t) \int_{0}^{t} U\left(t-t^{\prime}\right)\left\{\alpha(u v)\left(t^{\prime}\right)+\beta\left(|u|^{2} u\right)\left(t^{\prime}\right)\right\} d t^{\prime}, \\
& v(t)=\psi_{1}(t) V(t) v_{0}+\psi_{T}(t) \int_{0}^{t} V\left(t-t^{\prime}\right)\left\{\gamma \partial_{x}\left(|u|^{2}\right)\left(t^{\prime}\right)-\frac{1}{2} \partial_{x}\left(v^{2}\right)\left(t^{\prime}\right)\right\} d t^{\prime} .
\end{aligned}
$$

Solving the system (4.2) for all $t \in \mathbb{R}$ solves the system (4.1) locally in time for $|t| \leq T$, so that $T$ will be the time of local resolution of (4.1).

4.1. Proof of Theorem 1.1. We follow similar arguments as the ones given in [1] and $[9$.

We consider the following function space where we seek our solution:

$$
\Sigma_{\theta}:=\left\{(u, v) \in X^{k, \frac{1}{2}+\theta} \times Y^{s, \frac{1}{2}+\theta} ;\|u\|_{X^{k, 1 / 2+\theta}} \leq M_{1} \text { and }\|v\|_{Y^{s, 1 / 2+\theta}} \leq M_{2}\right\},
$$

where $0<\theta \ll 1$ and $M_{j}>0, j=1,2$, will be chosen below.

$\Sigma_{\theta}$ is a complete metric space with norm

$$
\|(u, v)\|_{\Sigma_{\theta}} \equiv\|u\|_{X^{k, 1 / 2+\theta}}+\|v\|_{Y^{s, 1 / 2+\theta}} .
$$


For $(u, v) \in \Sigma_{\theta}$ we define the maps

$$
\begin{aligned}
& \Phi_{1}(u, v)=\psi_{1}(t) U(t) u_{0}-i \psi_{T}(t) \int_{0}^{t} U\left(t-t^{\prime}\right)\left\{\alpha(u v)\left(t^{\prime}\right)+\beta\left(|u|^{2} u\right)\left(t^{\prime}\right)\right\} d t^{\prime}, \\
& \Phi_{2}(u, v)=\psi_{1}(t) V(t) v_{0}+\psi_{T}(t) \int_{0}^{t} V\left(t-t^{\prime}\right)\left\{\gamma \partial_{x}\left(|u|^{2}\right)\left(t^{\prime}\right)-\frac{1}{2} \partial_{x}\left(v^{2}\right)\left(t^{\prime}\right)\right\} d t^{\prime} .
\end{aligned}
$$

Let $a=\frac{1}{2}-2 \theta, b=\frac{1}{2}+\theta$ with $0<\theta \leq \tilde{\theta}(k, s)$ and $\tilde{\theta}(k, s)$ satisfy the following conditions:

$$
\tilde{\theta}(k, s)=\left\{\begin{array}{lll}
\min \left\{\frac{1}{12},-\frac{(2 s+1)}{2}, \frac{4 s+3}{12}\right\}, & \text { if } \quad(k, s) \in\left[0, \frac{1}{2}\right] \times\left(-\frac{3}{4},-\frac{1}{2}\right), \\
\frac{1}{24}-, & \text { if } \quad(k, s) \in\left[0, \frac{1}{2}\right] \times\left[-\frac{1}{2}, \infty\right), \\
\min \left\{\frac{1}{24}, \frac{2 k-1}{2}, \frac{2 k-2 s+1}{14}\right\}, & \text { if } \quad(k, s) \in\left(\frac{1}{2}, \infty\right) \times\left(-\frac{1}{2}, \frac{2 k+1}{2}\right) .
\end{array}\right.
$$

Then according to Lemma 2.1, with $b^{\prime}=-a$, and Lemmas 2.2 3.2 we have

$$
\begin{aligned}
\left\|\Phi_{1}(u, v)\right\|_{X^{k, 1 / 2+\theta}} & \leq c_{0}\left\|u_{0}\right\|_{H^{k}}+c_{1} T^{\theta}\left[\|u v\|_{X^{k,-1 / 2+2 \theta}}+\left\|\left.u\right|^{2} u\right\|_{X^{k,-1 / 2+2 \theta}}\right] \\
& \leq c_{0}\left\|u_{0}\right\|_{H^{k}}+c_{1} T^{\theta}\left[\|u\|_{X^{k, 1 / 2+\theta}}\|v\|_{Y^{s, 1 / 2+\theta}}+\|u\|_{X^{k, 1 / 2+\theta}}^{3}\right] \\
& \leq c_{0}\left\|u_{0}\right\|_{H^{k}}+c_{1} T^{\theta}\left[M_{1} M_{2}+M_{1}^{3}\right], \\
\left\|\Phi_{2}(u, v)\right\|_{Y^{s, 1 / 2+\theta}} & \leq c_{0}\left\|v_{0}\right\|_{H^{s}}+c_{2} T^{\theta}\left[\left\|\partial_{x} v^{2}\right\|_{Y^{s,-1 / 2+2 \theta}}+\left\|\partial_{x}|u|^{2}\right\|_{Y^{s,-1 / 2+2 \theta}}\right] \\
& \leq c_{0}\left\|v_{0}\right\|_{H^{s}}+c_{2} T^{\theta}\left[\|v\|_{Y^{s, 1 / 2+\theta}}^{2}+\|u\|_{X^{k, 1 / 2+\theta}}^{2}\right] \\
& \leq c_{0}\left\|v_{0}\right\|_{H^{s}}+c_{2} T^{\theta}\left[M_{1}^{2}+M_{2}^{2}\right] .
\end{aligned}
$$

Now taking $M_{1}=2 c_{0}\left\|u_{0}\right\|_{H^{k}}$ and $M_{2}=2 c_{0}\left\|v_{0}\right\|_{H^{s}}$ we have that

$$
\left\|\Phi_{1}(u, v)\right\|_{X^{k, 1 / 2+\theta}} \leq \frac{M_{1}}{2}+c_{1} T^{\theta}\left[M_{1}^{3}+M_{1} M_{2}\right]
$$

and

$$
\left\|\Phi_{2}(u, v)\right\|_{Y^{s, 1 / 2+\theta}} \leq \frac{M_{2}}{2}+c_{2} T^{\theta}\left[M_{1}^{2}+M_{2}^{2}\right] .
$$

Then $\left(\Phi_{1}(u, v), \Phi_{2}(u, v)\right) \in \Sigma_{\theta}$ for

$$
T^{\theta} \leq \frac{1}{2} \min \left\{\frac{1}{c_{1}\left[M_{1}^{2}+M_{2}\right]}, \frac{M_{2}}{c_{2}\left[M_{1}^{2}+M_{2}^{2}\right]}\right\} .
$$

Similarly we have that

$$
\begin{aligned}
\left\|\Phi_{1}(u, v)-\Phi_{1}(\tilde{u}, \tilde{v})\right\|_{X^{k, 1 / 2+\theta}} \leq & c_{3} T^{\theta}\left[M_{1}^{2}+M_{1}+M_{2}\right] \\
& \times\left[\|u-\tilde{u}\|_{X^{k, 1 / 2+\theta}}+\|v-\tilde{v}\|_{Y^{s, 1 / 2+\theta}}\right], \\
\left\|\Phi_{2}(u, v)-\Phi_{2}(\tilde{u}, \tilde{v})\right\|_{Y^{s, 1 / 2+\theta}} \leq & c_{4} T^{\theta}\left[M_{1}+M_{2}\right]\left[\|u-\tilde{u}\|_{X^{k, 1 / 2+\theta}}+\|v-\tilde{v}\|_{Y^{s, 1 / 2+\theta}}\right] .
\end{aligned}
$$

Then

$$
\left\|\left(\Phi_{1}(u, v), \Phi_{2}(u, v)\right)-\left(\Phi_{1}(\tilde{u}, \tilde{v}), \Phi_{2}(\tilde{u}, \tilde{v})\right)\right\|_{\Sigma_{\theta}} \leq \frac{1}{2}\|(u, v)-(\tilde{u}, \tilde{v})\|_{\Sigma_{\theta}}
$$

for

$$
T^{\theta} \leq \frac{1}{4} \min \left\{\frac{1}{c_{3}\left[M_{1}^{2}+M_{1}+M_{2}\right]}, \frac{1}{c_{4}\left[M_{1}+M_{2}\right]}\right\} .
$$

Therefore the map $\Phi_{1} \times \Phi_{2}: \Sigma_{\theta} \longrightarrow \Sigma_{\theta}$ is a contraction mapping, and we obtain a unique fixed point which solves the equation for any $T$ that satisfies (4.3) and (4.4). 


\section{Global solutions in the energy space $H^{1} \times H^{1}$}

In this section we prove Theorem 1.2 First we introduce the notation $\mathcal{M}_{0}=$ $\mathcal{M}(0), \mathcal{Q}_{0}=\mathcal{Q}(0)$ and $\mathcal{E}_{0}=\mathcal{E}(0)$, where $\mathcal{M}, \mathcal{Q}$ and $\mathcal{E}$ were defined in (1.2)-(1.4).

Let $\alpha \gamma>0$ and $t>0$. From (1.2) we have that $\|u(t)\|_{L^{2}}=\left\|u_{0}\right\|_{L^{2}}$, and from (1.3) we obtain

$$
\|v(t)\|_{L^{2}}^{2} \leq \frac{1}{|\alpha|}\left\{\left|\mathcal{Q}_{0}\right|+2|\gamma|\left\|u_{0}\right\|_{L^{2}}\left\|\partial_{x} u(t)\right\|_{L^{2}}\right\} .
$$

Let $\mu=\min \left\{|\gamma|, \frac{|\alpha|}{2}\right\}$. Then using (1.4), (5.1), and the Gagliardo-Nirenberg and Young inequalities we deduce

$$
\begin{aligned}
\left\|\partial_{x} u(t)\right\|_{L^{2}}^{2}+\left\|\partial_{x} v(t)\right\|_{L^{2}}^{2} \leq & \frac{1}{\mu}\left(|\gamma|\left\|\partial_{x} u(t)\right\|_{L^{2}}^{2}+|\alpha|\left\|\partial_{x} v(t)\right\|_{L^{2}}^{2}\right) \\
\leq & C\left(\left|\mathcal{E}_{0}\right|+\|v(t)\|_{L^{2}}\|u(t)\|_{L^{4}}^{2}+\|v(t)\|_{L^{3}}^{3}+\|u(t)\|_{L^{4}}^{4}\right) \\
\leq & C\left(\left|\mathcal{E}_{0}\right|+\|v(t)\|_{L^{2}}^{2}+\|v(t)\|_{L^{3}}^{3}+\|u(t)\|_{L^{4}}^{4}\right) \\
\leq & C\left(\left|\mathcal{E}_{0}\right|+\left|\mathcal{Q}_{0}\right|+\left\|u_{0}\right\|_{L^{2}}\left\|\partial_{x} u(t)\right\|_{L^{2}}+\|v(t)\|_{L^{3}}^{3}+\|u(t)\|_{L^{4}}^{4}\right) \\
\leq & C\left\{\left|\mathcal{E}_{0}\right|+\left|\mathcal{Q}_{0}\right|+\left|\mathcal{Q}_{0}\right|^{\frac{5}{3}}+\mathcal{M}_{0}^{5}+\mathcal{M}_{0}^{3}+\mathcal{M}_{0}\right\} \\
& +\frac{1}{2}\left\{\left\|\partial_{x} u(t)\right\|_{L^{2}}^{2}+\left\|\partial_{x} v(t)\right\|_{L^{2}}^{2}\right\} .
\end{aligned}
$$

Hence

$$
\left\|\partial_{x} u(t)\right\|_{L^{2}}^{2}+\left\|\partial_{x} v(t)\right\|_{L^{2}}^{2} \leq C\left\{\left|\mathcal{E}_{0}\right|+\left|\mathcal{Q}_{0}\right|+\left|\mathcal{Q}_{0}\right|^{\frac{5}{3}}+\mathcal{M}_{0}^{5}+\mathcal{M}_{0}^{3}+\mathcal{M}_{0}\right\} .
$$

We can estimate the right hand side of (5.3) using the conservation laws (1.3) and (1.4) and Sobolev's lemma to get

$$
\|u(t)\|_{H^{1}}^{2}+\|v(t)\|_{H^{1}}^{2} \leq \Psi\left(\left\|u_{0}\right\|_{H^{1}},\left\|v_{0}\right\|_{H^{1}}\right),
$$

where $\Psi$ is a function depending only on $\left\|u_{0}\right\|_{H^{1}}$ and $\left\|v_{0}\right\|_{H^{1}}$.

We observe that the constants depend only on the parameters $\alpha, \beta$ and $\gamma$.

Since the right hand side of (5.4) only depends of $\left\|u_{0}\right\|_{H^{1}}$ and $\left\|v_{0}\right\|_{H^{1}}$, we can repeat the argument of local existence of solution at time $T$ arriving at a solution for any positive time. The same holds for negative time.

\section{ACKNOWLEDGMEnTs}

The first author would like to thank CNPq-Brazil for the financial support to complete his Ph.D. Most of the content of this paper is part of his Ph.D. Thesis ([7]).

\section{REFERENCES}

[1] D. Bekiranov, T. Ogawa and G. Ponce, Weak solvability and well-posedness of a coupled Schrödinger-Korteweg de Vries equation for capillary-gravity wave interactions, Proceedings of the AMS., 125 (10), (1997), 2907-2919. MR.1403113 (97m:35238)

[2] D. Bekiranov, T. Ogawa and G. Ponce, Interaction equations for short and long dispersive waves, J. Funct. Anal., 158, (1998), 357-388. MR1648479 (99i:35143)

[3] E. S. Benilov and S. P. Burtsev, To the integrability of the equations describing the Langmuir-wave-ion-acoustic-wave interaction, Phys. Let., 98A (1983), 256-258. MR0720816 (85f:76120) 
[4] J. Bourgain, Fourier transform restriction phenomena for certain lattice subsets and applications to nonlinear evolution equations, Geometric and Functional Anal., 3 (1993), 107-156, 209-262. MR.1209299 (95d:35160a) MR 1215780(95d:35160b)

[5] M. Christ, J. Colliander, and T. Tao, Asymptotics, frequency modulation, and low regularity ill-posedness for canonical defocusing equations, Amer. J. Math., 125 (2003), no. 6, 12351293. MR2018661 (2005d:35223)

[6] M. Christ, J. Colliander, and T. Tao, Ill-posedness for nonlinear Schrödinger and Wave equations, preprint.

[7] A. J. Corcho, On Some Nonlinear Dispersive Systems, Ph.D. Thesis. Informes de Matemática. IMPA, Rio de Janeiro, 18 (2003).

[8] M. Funakoshi and M. Oikawa, The resonant interaction between a long internal gravity wave and a surface gravity wave packet, J. Phys. Soc. Japan, 52 (1983), 1982-1995. MR0710730 (84k:76030)

[9] J. Ginibre, Y.Tsutsumi and G. Velo, On the Cauchy Problem for the Zakharov system, J. Funct. Anal., 151 (1997), 384-436. MR.1491547 (2000c:35220)

[10] B. Guo and Ch. Miao, Well-posedness of the Cauchy problem for the coupled system of the Schrödinger-KdV equations, Acta Math. Sinica, Engl. Series, 15 (1999), 215-224. MR1714079 (2000e:35207)

[11] H. Hojo, H. Ikezi, K. Mima and K. Nishikawa, Coupled nonlinear electron-plasma and ionacoustic waves, Phys. Rev. Lett., 33 (1974), 148-151.

[12] T. Kakutani, T. Kawahara and N. Sugimoto, Nonlinear interaction between short and long capillary-gravity waves, J. Phys. Soc. Japan, 39 (1975), 1379-1386.

[13] C. E. Kenig, G. Ponce and L. Vega, The Cauchy problem for the Korteweg-de Vries equation in Sobolev spaces of negative indices, Duke Math. J., 71 (1993), 1-21. MR1230283 (94g:35196)

[14] C. E. Kenig, G. Ponce and L. Vega, A bilinear estimate with applications to the KdV equation, J. Amer. Math. Soc., 9 (1996), 573-603. MR.1329387 (96k:35159)

[15] C. E. Kenig, G. Ponce and L. Vega, Quadratic Forms for the 1-D semilinear Schrödinger equation, Trans. Amer. Math. Soc., 348 (1996), no. 8, 3323-3353. MR1357398 (96j:35233)

[16] C. E. Kenig, G. Ponce and L. Vega, On ill-posedness of some canonical dispersive equations, Duke Math. J., 106 (2001), 617-633. MR1813239 (2002c:35265)

[17] J. Satsuma and N. Yajima, Soliton solutions in a diatomic lattice system, Progr. Theor. Phys., 62 (1979), 370-378.

[18] Y. Tsutsumi, $L^{2}$-solutions for nonlinear Schrödinger equations and nonlinear groups, Funkcialaj Ekvacioj, 30 (1987), 115-125. MR0915266 (89c:35143)

[19] M. Tsutsumi, Well-posedness of the Cauchy problem for a coupled Schrödinger-KdV equation, Math. Sciences Appl., 2 (1993), 513-528. MR1370488 (96k:35163)

Departamento de Matemática, Universidade Federal de Alagoas, Campus A. C. Simões, Tabuleiro dos Martins, Maceió-AL, 57072-970, Brazil

E-mail address: adan@mat.ufal.br

impa, Estrada Dona Castorina 110, Rio de Janeiro, 22460-320, Brazil

E-mail address: linares@impa.br 